\title{
Mild Sensory Stimulation Reestablishes Cortical Function during the Acute Phase of Ischemia
}

\author{
Christopher C. Lay, ${ }^{1,3}$ Melissa F. Davis, ${ }^{1,3}$ Cynthia H. Chen-Bee, ${ }^{1}$ and Ron D. Frostig ${ }^{1,2,3}$ \\ ${ }^{1}$ Department of Neurobiology and Behavior, ${ }^{2}$ Department of Biomedical Engineering, and ${ }^{3}$ The Center for Learning and Memory, University of California, \\ Irvine, California 92697-4550
}

When delivered within 1 and in most cases $2 \mathrm{~h}$ of permanent middle cerebral artery occlusion (pMCA0), mild sensory stimulation (intermittent single whisker stimulation) was shown to be completely neuroprotective $24 \mathrm{~h}$ after pMCAO in a rodent model of ischemic stroke, according to assessment with multiple techniques (Lay et al., 2010). The acute effect of stimulation treatment on the ischemic cortex, however, has yet to be reported. Here we characterize cortical function and perfusion during the 120 min whisker stimulation period in four experimental groups with treatment initiated $0,1,2$ (protected groups), or $3 \mathrm{~h}$ (unprotected group) post-pMCA0 using multiple techniques. According to functional imaging, a gradual return of evoked whisker functional representation to baseline levels was initiated with treatment onset and completed within the treatment period. Evoked neuronal activity and reperfusion to the ischemic area also showed a gradual recovery in protected animals. Surprisingly, a similar recovery profile was observed in response to treatment in all protected animals, regardless of treatment onset time. Nonstimulated pMCAO control group data demonstrate that reperfusion is not spontaneous. This makes the complete protection observed in the majority of animals stimulated at $2 \mathrm{~h}$ post-pMCA0 even more surprising, as these animals recovered despite having been in a severely ischemic state for two full hours. In summary, when delivered within a $2 \mathrm{~h}$ window post-pMCA0, whisker stimulation treatment initiated reperfusion and a gradual recovery of cortical function that was completed or nearly completed within the treatment period.

\section{Introduction}

Middle cerebral artery (MCA) occlusion is used to model the most clinically relevant type and location of stroke in humans: ischemic stroke within MCA (Lloyd-Jones et al., 2009). Experimental MCA occlusions also lead to loss of function, cortical infarct, and behavioral impairment (Wang-Fischer, 2009). We have previously shown that, if initiated within 1 and in most cases $2 \mathrm{~h}$ of permanent middle cerebral artery occlusion (pMCAO) in a rat model, intermittent single whisker stimulation prevents the expected damage and deficits. This protective stimulation treatment consisted of 4.27 min of $5 \mathrm{~Hz}$ single whisker stimulation delivered over $120 \mathrm{~min}$ (for details, see Lay et al., 2010). Subjects were assessed at $24 \mathrm{~h}$ post-pMCAO using intrinsic signal optical imaging (ISOI), extracellular recording, and laser speckle blood flow imaging (LSI). In another set of animals, behavior was assessed 1 week post-pMCAO. Postmortem histological analysis

\footnotetext{
Received April 6, 2011; revised June 10, 2011; accepted June 15, 2011.

Author contributions: C.C.L., M.F.D., C.H.C.-B., and R.D.F. designed research; C.C.L. and M.F.D. performed research; C.C.L., M.F.D., and C.H.C.-B. analyzed data; C.C.L., M.F.D., C.H.C.-B., and R.D.F. wrote the paper.

This work was supported by the American Heart Association Predoctoral Fellowship 788808-41910 and the NIH-NINDS NS-066001 and NS-055832. The funders had no role in study design, data collection and analysis, decision to publish, or preparation of the manuscript. We thank Dr. David Abookasis for his input during the early stages of this project. We thank Q. Vu, K. Julian, T. Truong, and A. Nierode for their assistance with imaging and histology.

${ }^{*}$ C.C.L. and M.F.D. contributed equally to this work.

Correspondence should be addressed to Dr. Ron Frostig, Departments of Neurobiology and Behavior, and Biomedical Engineering, Fellow, Center for the Neurobiology of Learning and Memory, 2205 McGaugh Hall, University of California, Irvine, Irvine, CA 92697. E-mail: rfrostig@uci.edu.

DOI:10.1523/JNEUROSCI.1741-11.2011

Copyright $\odot 2011$ the authors $\quad 0270-6474 / 11 / 3111495-10 \$ 15.00 / 0$
}

[2,3,5-triphenyltetrazolium chloride (TTC) staining] was performed in all cases (Lay et al., 2010). Animals that received early stimulation treatment had pre-pMCAO baseline level results according to all of the above methods and showed no sign of infarct. In fact, altering the pattern and distribution of this whisker stimulation did not have an effect on its ability to protect (Davis et al., 2011). In contrast, however, animals that did not receive treatment until $3 \mathrm{~h}$ post-pMCAO had eliminated function and sustained large infarcts. Blood flow data suggested that the protection induced by early stimulation was due to reorganized blood flow via collateral vessels (interarterial connections), which have recently been suggested to be an important potential avenue for future stroke therapy (Schaffer et al., 2006; Armitage et al., 2010). The stimulation treatment induced complete protection from ischemic stroke by $24 \mathrm{~h}$ post pMCAO, but cortical function during the acute phase of ischemia over the treatment period was not assessed. Thus, the presumed initial loss of function following pMCAO and the recovery induced by the treatment had yet to be investigated. In the current study, using the previous study's pMCAO model and whisker stimulation treatment, animals were assessed using ISOI, extracellular recording, or LSI at four evenly spaced time points over the 120 min treatment period.

Using ISOI, we observed an initial (post-pMCAO) absent or severely diminished whisker functional representation followed by gradual recovery to baseline responses over the treatment period. Surprisingly, the observed time course of recovery was the same whether treatment began 0,1 , or $2 \mathrm{~h}$ post-pMCAO. When treatment was delivered $3 \mathrm{~h}$ post-pMCAO, however, animals 
never showed signs of returning function. Evoked subthreshold activity, evoked spiking, and blood flow levels were also severely decreased or eliminated immediately postocclusion and returned gradually to preocclusion or near-preocclusion levels by the completion of the stimulation treatment period in protected animals.

Together, these findings demonstrate that recovery to preocclusion levels is initiated and completed or nearly completed during the 120 min period of protective stimulation itself, regardless of the timing of stimulus delivery onset within the $2 \mathrm{~h}$ window of opportunity for protection. It has been suggested that there is a time window for neuroplasticity following stroke onset during which impending damage can be reduced (Murphy and Corbett, 2009). Our data suggest that this window of opportunity extends additionally to neurovascular changes that can facilitate full protection.

Understanding these parameters of functional recovery in response to stimulation might lead to diagnostically relevant means of guiding patient care during the initial hours of ischemia, should this treatment prove translational.

\section{Materials and Methods}

All procedures were in compliance with NIH guidelines and approved by University of California Irvine Animal Care and Use Committee.

Subjects and surgical preparation. Experimental subjects (295-400 g male Sprague Dawley rats) were individually housed in standard cages. At the beginning of each experiment, animals were injected intraperitoneally with a Nembutal bolus [ $55 \mathrm{mg} / \mathrm{kg}$ body weight (b.w.)]. Supplemental injections of Nembutal ( $27.5 \mathrm{mg} / \mathrm{kg}$ b.w.) were given as necessary. After resection of soft tissue, an $\sim 6.5 \times \sim 8 \mathrm{~mm}$ imaging area of the skull over the left and right primary somatosensory cortex (rostromedial corner positioned $\sim 1 \mathrm{~mm}$ caudal and $2 \mathrm{~mm}$ lateral from bregma) was thinned to $\sim 150 \mu \mathrm{m}$ using a dental drill. Five percent dextrose $(3 \mathrm{ml})$ and atropine $(0.05 \mathrm{mg} / \mathrm{kg}$ b.w. $)$ were administered at the beginning of the experiment and every $6 \mathrm{~h}$ after until the experiment was complete. Body temperature was measured via a rectal probe, and maintained at $37^{\circ} \mathrm{C}$ by a self-regulating thermal blanket.

Overview. Following baseline assessment, all rats underwent pMCAO and were then randomly assigned to one of the following experimental groups: $+0 \mathrm{~h},+1 \mathrm{~h},+2 \mathrm{~h}$, or $+3 \mathrm{~h}$. Groups were so named for the latency (in hours) between pMCAO and the initiation of postocclusion whisker stimulation ( $+0 \mathrm{~h}, 0 \mathrm{~h}$ between pMCAO and initiation of treatment; +1 $\mathrm{h}, 1 \mathrm{~h}$ between pMCAO and initiation of treatment, and so on). Two groups of protected animals were also assessed for recovery of neuronal function or blood flow. Following baseline assessment for the extracellular recording and LSI, all rats also underwent pMCAO and immediate stimulation treatment ( $+0 \mathrm{~h}$ group). Postocclusion whisker stimulation treatment consisted of 256, $1 \mathrm{~s}, 5 \mathrm{~Hz}$ deflections of a single whisker (whisker C2) delivered intermittently (with random intervals averaging $21 \mathrm{~s}$ ), resulting in a total of $4.27 \mathrm{~min}$ of stimulation over the course of 120 $\mathrm{min}$. In addition to acting as treatment, stimulation evoked the responses measured in functional and neuronal recording assessment. Data were collected at four time points (every $30 \mathrm{~min}$ ) during the $120 \mathrm{~min}$ postpMCAO whisker stimulation treatment period regardless of technique or experimental group. Following whisker stimulation treatment, animals were returned to their home cages and allowed to recover. All animals were killed $24 \mathrm{~h}$ after pMCAO and TTC staining was used to verify the expected presence or absence of infarct based on the results of the previous study (Lay et al., 2010).

PMCAO. Ischemic conditions were achieved via surgical occlusion and transection of the M1 segment (just distal to lenticulostriate branching) of the left middle cerebral artery such that only MCA cortical branches were affected and thus only cortical infarct (no subcortical damage) was expected (Tamura et al., 1981; Brint et al., 1988; WangFischer, 2009). The skull and dura were carefully removed from a $2 \times 2$ $\mathrm{mm}$ surgical window just anterior and lateral to the imaging window (over the occlusion location; the M1 segment just distal to MCA's lenticulostriate branching) and a half-curve reverse cutting suture needle and thread (4-0 silk) was passed through the pial layer of the meninges, below MCA and above the cortical surface. A double ligature was tied and tightened around MCA and the vessel was then transected (completely severed) between the two knots. Experiments were terminated if there was any sign of bleeding from MCA or if there were obvious arterial abnormalities or malformations (Fox et al., 1993; Niiro et al., 1996).

Histology (TTC staining for infarct). Rats were killed with $3.0 \mathrm{ml}$ of Euthasol at the conclusion of each experiment. Their brains were carefully removed and sectioned into $2 \mathrm{~mm}$ slices along the coronal plane. The brain slices were then incubated in $2 \%$ TTC at $37^{\circ} \mathrm{C}$ for $20 \mathrm{~min}$ in the dark (Bederson et al., 1986). TTC is enzymatically reduced, producing formazan (a bright red byproduct) by dehydrogenases in active mitochondria. Red stain intensity correlates with the number and functional activity of mitochondria, with unstained (white) areas being indicative of infarct (Goldlust et al., 1996; Lavie et al., 2001). The TTC-stained sections were photographed with a digital camera and the total infarct volume was determined by multiplying the infarct area of each slice by the thickness of that slice. An observer blind to experimental condition performed this volume calculation. A small lesion $(\sim 1 \mathrm{~mm}$ in diameter or less) was occasionally apparent at the immediate site of MCA occlusion. This occurred infrequently and equivalently in all experimental groups (1-2 rats per group). The small amount of damage occasionally produced at the surgical site could be readily distinguished from the large ischemic infarct and was excluded from infarct analysis (Tamura et al., 1981).

ISOI and analysis. We used the functional imaging technique ISOI to assess whisker functional representation (WFR; intrinsic signal evoked by the stimulation of a single whisker) before pMCAO and within the ischemic region at each of four post-pMCAO time points (spaced $\sim 30$ min apart) across the 120 min stimulation treatment period. To verify that changes were limited to the ischemic cortex, we also assessed cortical function in the contra-ischemic hemisphere before and following treatment. ISOI allows high-resolution characterization of WFRs in response to sensory stimulation (for review, see Frostig and Chen-Bee, 2009). A detailed description of ISOI (Grinvald et al., 1986; Frostig et al., 1990; Ts'o et al., 1990) data acquisition and analysis can be found previously (Chen-Bee et al., 2000, 2007). Briefly, a charge-coupled device (CCD) camera (either a 16-bit Cascade 512F or a 12-bit Quantix 0206) equipped with an inverted $50 \mathrm{~mm}$ AF Nikon lens (1:1:8) combined with an extender (model PK-13) was used for imaging and controlled by $\mathrm{V}++$ Precision Digital Imaging System software (Digital Optics). The cortex was illuminated with a red light emitting diode ( $635 \mathrm{~nm}$ maximum wavelength with full-width at half-height of $15 \mathrm{~nm}$ ). During each $15 \mathrm{~s}$ trial, $1.5 \mathrm{~s}$ of prestimulus data followed by $13.5 \mathrm{~s}$ of poststimulus-onset data were collected, with a $6 \pm 5 \mathrm{~s}$ random intertrial interval (total time between one trial onset and the next was therefore $21 \mathrm{~s}$ on average). Stimulus consisted of a single whisker (whisker C2) being deflected by $\sim 9^{\circ}$ in the rostral-caudal direction at a rate of $5 \mathrm{~Hz}$ for total stimulus duration of $1 \mathrm{~s}$. This same stimulation protocol was also used to evoke response in extracellular recording and laser speckle blood flow imaging experiments. Data were collected in four blocks of 64 stimulation trials over a period of $\sim 30 \mathrm{~min}$ each. Ratio images were created from calculating fractional change values for each of the four 64 trial blocks by dividing each $500 \mathrm{~ms}$ frame of poststimulus signal activity by the $500 \mathrm{~ms}$ frame of prestimulus intrinsic signal activity collected immediately before stimulus onset. The first two phases of whisker functional representation evoked by single whisker stimulation were analyzed. These included the initial dip (the first phase generally associated with evoked neuronal activity) and the overshoot (the second phase, generally associated with blood flow change initiated by the activity). The ratio image containing the maximum areal extent for each of the two phases was Gaussian filtered (half-width $=5$ ) and the areal extent quantified at a threshold level of $2.5 \times 10^{-4}$ away from zero. Peak amplitude was quantified in fractional change units from the pixel with the peak activity within the maximum areal extent for each of the two intrinsic signal phases.

Electrophysiology: extracellular recording and analysis. Before pMCAO and extracellular recording, ISOI was performed to identify the location of peak optical activity evoked by whisker $\mathrm{C} 2$ stimulation to guide proper placement of electrodes for subsequent neuronal recording (Masino et al., 1993; Brett-Green et al., 2001; Frostig et al., 2008). Electrophysiology 
animals underwent the same initial experimental protocol as ISOI animals, with data collected before pMCAO and at each of the same four post-pMCAO time points across the 120 min treatment period. Following ISOI mapping, the thinned skull region and underlying dura were removed, the cisterna magnum was drained of CSFs to minimize edema, and the exposed cortex was covered with silicon oil. A Tungsten microelectrode $(\sim 1.5 \mathrm{~m} \Omega)$ was inserted into the exposed cortex such that the electrode entered perpendicular to the location of peak optical activity as determined using ISOI. We used data acquisition hardware and software from Alpha-Omega. Simultaneous recordings of both suprathreshold [multiunit activity (MUA)] and subthreshold [local field potential (LFP)]-evoked neuronal activity were obtained from a depth of $\sim 300$ $400 \mu \mathrm{m}$ (supragranular layer) below the cortical surface. Depth was determined while penetrating the cortex using a micropositioner with $1 \mu \mathrm{m}$ resolution steps. Recorded signals were amplified and bandpass (1-3000 $\mathrm{Hz}$ ) filtered to allow simultaneous capture of MUA and LFP from the same electrode, and then digitized at $24 \mathrm{kHz}$ rate. Real-time traces and multiunit peristimulus time histograms (PSTH) were generated to monitor quality and consistency of recordings. As mentioned, recording sessions consisted of the same whisker stimulation parameters used during ISOI. Spike2 software (Cambridge Electronic Design) was used for the off-line extraction of MUA and LFP by refiltering the collected data in either the $1-300 \mathrm{~Hz}$ (LFP) or $300-3000 \mathrm{~Hz}$ (MUA) range for the subsequent analysis. A single average LFP waveform or MUA PSTH in $1 \mathrm{~ms}$ bins was generated from 64 stimulation trials. LFP magnitude was then calculated as the mean of all five peak on responses minus the mean obtained from $1 \mathrm{~s}$ duration of prestimulus data. Similarly, MUA magnitude was calculated as the mean firing rate (spikes/s) of all five peak on responses minus the mean firing rate obtained from $1 \mathrm{~s}$ duration of prestimulus data. Additionally, during the $1 \mathrm{~s}$ prestimulus period, spontaneous activity was recorded. The same threshold within animals was used to qualify both evoked and spontaneous spiking events.

LSI of blood flow and analysis. A detailed description of LSI (Dunn et al., 2001; Choi et al., 2006) data acquisition and analysis can be found previously (Lay et al., 2010).

Briefly, a $632.8 \mathrm{~nm} 15 \mathrm{~mW} \mathrm{HeNe}$ laser was used as the illumination source. The laser beam was first expanded with a $2 \times$ lens to illuminate the thinned skull region of $\sim 25 \mathrm{~mm}^{2}$ in a more uniform manner. Care was taken to maintain the same level of illumination intensity over the imaged area of interest within each experiment. The speckle pattern from the $5.12 \times 5.12 \mathrm{~mm}$ imaged region was captured as $512 \times 512$ pixel images by a 16-bit CCD camera (Cascade 512 F) equipped with a Navitar zoom lens plus extenders such that speckle size matched camera pixel size. The frame exposure time for each image was $1 \mathrm{~ms}$, and 10 consecutive images spaced $1.5 \mathrm{~s}$ apart were collected per time point. Collected images were processed as previously described (Briers and Webster, 1995). Briefly, for each time point, the 10 raw speckle images were converted to speckle contrast images by calculating the coefficient of variation $(\mathrm{SD} /$ mean $)$ for the center pixel within $5 \times 5$ pixel sliding windows in each image. The resulting 10 speckle contrast images were then converted to speckle index images by calculating their inverse squares multiplied by the exposure time in seconds, so that larger index values corresponded to faster blood flow. Lastly, the 10 speckle index images of each sampled time point were averaged to improve signal-to-noise ratio. LSI animals underwent the same initial experimental protocol as ISOI animals with data collected before pMCAO and at each of the same four post-pMCAO time points across the 120 min period post-pMCAO. For each data collection time point, 10 raw speckle images were collected at $1 \mathrm{~s}$ intervals with $1 \mathrm{~ms}$ exposure times and converted to speckle contrast images. The resulting 10 speckle contrast images were then converted to speckle index images, so that larger index values corresponded to faster blood flow. Last, the 10 speckle index images of each sampled time point were averaged to improve signal-to-noise ratio.

To quantify blood flow within specific MCA branches downstream from the occlusion for each sampled time point, we calculated the mean value within a region of interest (ROI) in MCA cortical branches as defined according to several criteria described previously (Lay et al., 2010).

To determine noise level values, we also imaged a subset of killed animals at $5 \mathrm{~min}$ after the cessation of heart beat and averaged these values collected from the nonflowing MCA (termed noise or no-flow values). Analysis of an MCA ROI was performed for these animals in the same manner as for live animals.

Finally, all flow index values were scaled over a range where 0 flow was set at noise values (the average flow value collected from an MCA region of interest in the cortices of dead animals in the same room using the same set-up). A between-subjects repeated-measures analysis was then performed on +0 h subjects versus no-stimulus control subjects (animals that had undergone pMCAO but never received stimulation treatment) to determine the degree of spontaneous reperfusion in the $0-120$ min period post-pMCAO independent of stimulation versus the degree of stimulation-induced reperfusion during the $120 \mathrm{~min}$ treatment period in +0 h subjects.

\section{Statistical analysis}

For statistical analysis, within-subjects repeated-measures ANOVAs were used with an $\alpha$-level of significance set at 0.05 . ISOI data collected within the ischemic hemisphere were converted to difference score values (postocclusion - baseline), a constant was added to each difference score, and each score was then transformed with a square root function to better satisfy the assumptions of an ANOVA (normal distribution for each subgroup of values, homogeneity of variance across means, independence between variance and means). Raw values of subthreshold, suprathreshold, and laser speckle velocity were used for analysis. For all data, post hoc contrasts were performed to identify which postocclusion values were significantly different from baseline. Alpha level was set to 0.05 and Bonferroni adjustments were applied to account for multiple contrasts.

\section{Results}

pMCAO results in a severe loss of perfusion and late administration of treatment ( $3 \mathrm{~h}$ post-pMCAO) results in an irreversible loss of function and ischemic injury

To ensure that pMCAO was successful, we first assessed blood flow within MCA before and immediately following occlusion using LSI within cortical branches of MCA ( $n=7$ animals; for details, see Materials and Methods, above). According to LSI, perfusion in MCA dropped to levels approaching noise and was significantly below baseline (paired $t$ test, $t_{(6)}=8.63, p<$ 0.001) immediately following pMCAO (for representative case, see Fig. $1 A$ ).

We also examined cortical function during late stimulation treatment (delivered too late to protect from ischemic damage; 3 h post-pMCAO: $+3 \mathrm{~h}, n=10$ ) [previous research in our lab has shown that $+3 \mathrm{~h}$ animals have no sign of evoked functional activity at $24 \mathrm{~h}$ postocclusion and sustain large cortical infarcts (Lay et al., 2010)]. In the current study, we examined functional activity during the treatment period itself. $+3 \mathrm{~h}$ animals' imaging data were collected at baseline and every $30 \mathrm{~min}$ throughout the 120 min treatment period (that began $3 \mathrm{~h}$ after pMCAO in these animals). We observed a complete lack of evoked cortical function in all $+3 \mathrm{~h}$ animals at every recorded time point during the treatment period (Fig. $1 B, D$ ). All $+3 \mathrm{~h}$ animals also sustained the expected large cortical infarct when assessed the following day (range, 30.4-82.6 $\mathrm{mm}^{3}$; mean, $59.2 \pm 4.4 \mathrm{~mm}^{3}$ ) (for a representative case, see Fig. 1C).

\section{ISOI overview: when whisker stimulation treatment is delivered within $2 \mathrm{~h}$ of ischemic onset, it initiates a gradual return to baseline level function within the treatment period}

As the primary focus of this study was to assess the recovery of function during the treatment period in protected animals, we conducted functional imaging in three protected groups with varying treatment onset times: treatment initiated immediately $(+0 \mathrm{~h}, n=10), 1 \mathrm{~h}(+1 \mathrm{~h}, n=10)$, or $2 \mathrm{~h}(+2 \mathrm{~h}, n=10)$ 

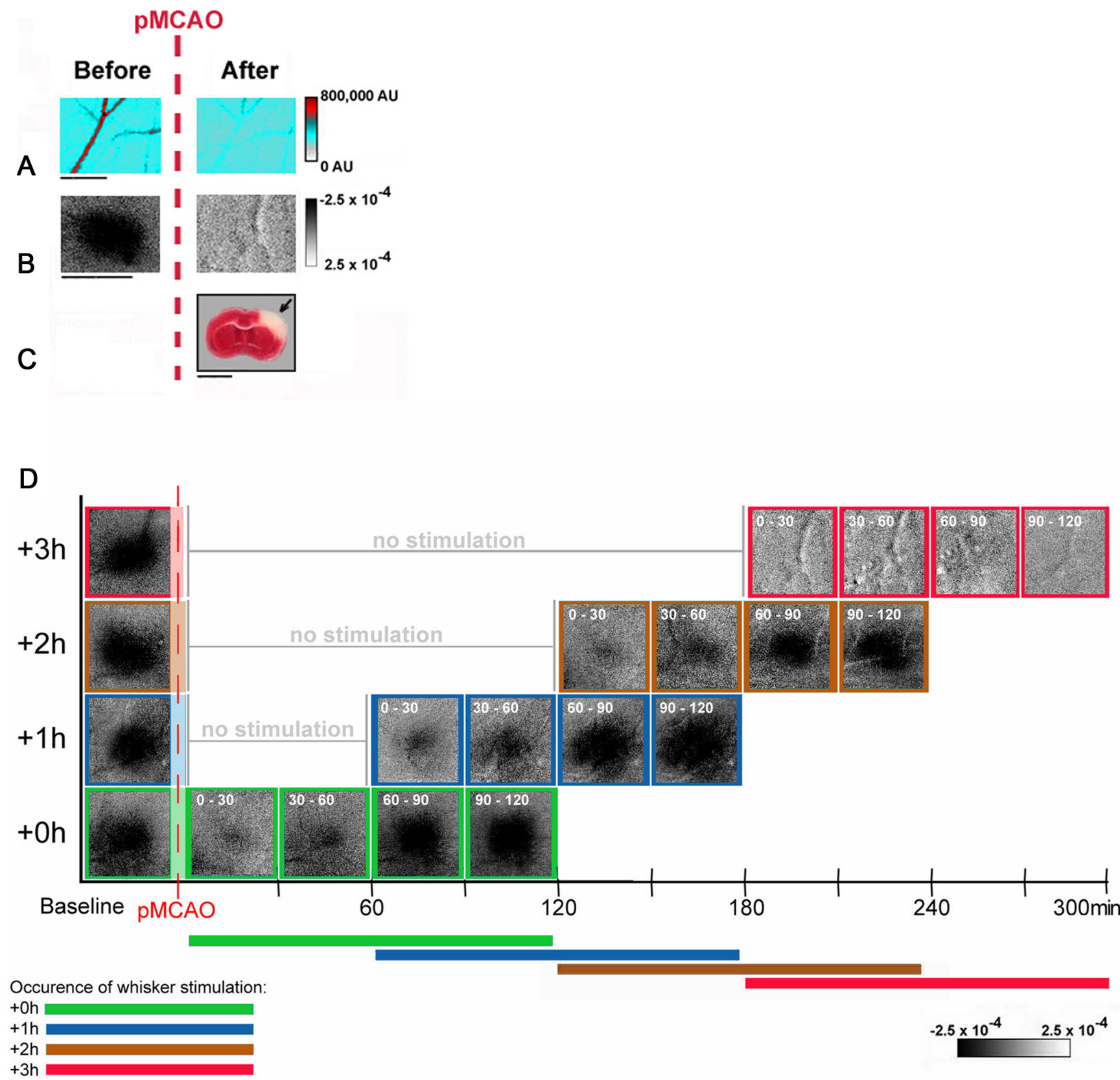

Figure 1. pMCA0 produces irreversible damage in animals that receive treatment $3 \mathrm{~h}$ postocclusion ( $+3 \mathrm{~h}$ animals), while return of cortical function is evident in $+0 \mathrm{~h},+1 \mathrm{~h}$, and $+2 \mathrm{~h}$ groups during protective stimulation treatment. Data is from representative animals that underwent PMCAO and received whisker stimulation treatment $3 \mathrm{~h}$ postocclusion. $\mathrm{A}$, Linearly color-scaled LSI images taken at baseline and following pMCAO in a $+3 \mathrm{~h}$ animal. The vessel traversing these images is a representative segment of the analyzed MCA ROl in a branch of MCA above somatosensory cortex, distal to pMCAO. Scale bar, $1 \mathrm{~mm}$. B, Intrinsic signal optical imaging was conducted before pMCAO and during the postocclusion treatment period. Whisker functional representation was completely absent throughout the entire postocclusion stimulus period in $+3 \mathrm{~h}$ animals. Linear grayscale bar indicates intrinsic signal strength $\times 10^{-4}$. Black and white streaks correspond to large surface blood vessels. Scale bar, $5 \mathrm{~mm}$. C, Coronal section taken from TTC assay for infarct in a $+3 \mathrm{~h}$ animal. Note that the area devoid of staining (arrow) within the $+3 \mathrm{~h}$ subject's cortex indicates ischemic infarct due to pMCAO and late stimulation treatment. Scale bar, $5 \mathrm{~mm}$. D, Representative data from intrinsic signal optical imaging of the initial dip for each group $(+0 \mathrm{~h},+1 \mathrm{~h},+2 \mathrm{~h}$, and $+3 \mathrm{~h}$ ) were arranged according to time of treatment initiation for comparison (treatment time is included in white in the top left corner of each image). Groups $+0 \mathrm{~h},+1 \mathrm{~h}$, and $+2 \mathrm{~h}$ all regained evoked functional response comparable to baseline after $90 \mathrm{~min}$ of whisker stimulation, while $+3 \mathrm{~h}$ animals never demonstrated any post-pMCA0 cortical activity.

post-pMCAO. The general experimental scheme for assessment of whisker functional representation before, during, and after pMCAO is summarized in Fig. $1 D$ for all experimental groups. In addition, we conducted functional imaging in a sham operation (sham-pMCAO; $n=10$ ) group to determine the level of variation in functional activity over the course of the stimulation period. All animals were assessed at baseline and at four time points (spaced $\sim 30$ min apart) across the 120 min treatment period. In sham-pMCAO animals, there were no significant fluctuations in either the initial dip (area, $F_{(4,24)}=0.59, p>0.05$; amplitude, $F_{(4,24)}=0.84, p>0.05$; ANOVAs) or the overshoot (area, $F_{(4,24)}=0.16, p>0.05$; amplitude, $F_{(4,24)}=0.60, p>0.05$; ANOVAs) throughout experimentation (Fig. 2). In contrast, both protected $(+0 \mathrm{~h},+1 \mathrm{~h},+2 \mathrm{~h})$ and unprotected $(+3 \mathrm{~h})$ animals exhibited a profound loss of WFR during the first data collection and stimulation block post-pMCAO. WFR returned and increased over the following data collection blocks in protected animals, but unprotected animals continued to have a lack of re- 

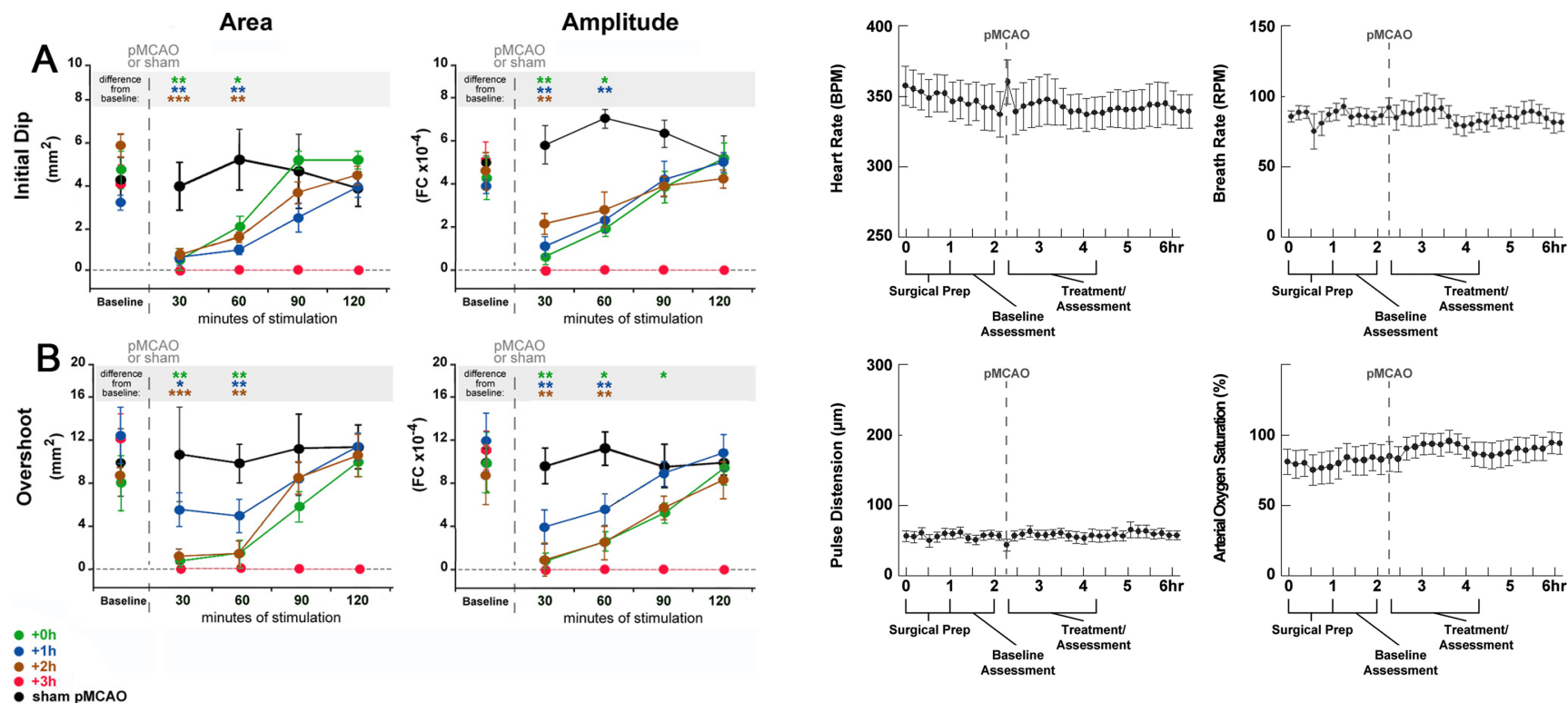

Figure 2. Whisker functional representation returns gradually upon stimulation onset in all protected animals throughout the treatment period. $\boldsymbol{A}, \boldsymbol{B}$, Group baseline is plotted with $120 \mathrm{~min}$ of postocclusion stimulation period data. Means and SEs are provided for the area (left) and amplitude (right) of the ipsi-ischemic $(2$ initial dip ( $\boldsymbol{A}$ ) and overshoot ( $\boldsymbol{B})$ phases of whisker functional representation before and after pMCA0. $+3 \mathrm{~h}$ animals had no response to stimulation at any time point post-pMCA0. For all other groups (shampMCA0, $+0 \mathrm{~h},+1 \mathrm{~h}$, and $+2 \mathrm{~h}$ ), asterisks indicate a significant difference from baseline: ${ }^{*} p<0.05,{ }^{* *} p<0.01$, and ${ }^{* * *} p<0.001$.

sponse throughout all collection blocks. Thus, return of whisker functional representation or lack thereof during stimulation treatment was predictive of final outcome.

Return of function was quantified in greater detail by examining the area and amplitude of the first two phases of whisker functional representation: the initial dip and the overshoot (Chen-Bee et al., 1996, 2007). The initial dip and overshoot phases observed in functional imaging techniques are thought to result from underlying hemodynamic processes that act as proxies for neuronal activity. The exact sources of the different phases are still debated, but generally the initial dip is better correlated with suprathreshold activation and deoxygenation while blood flow volume changes and oxygenated blood flow are thought to underlie the overshoot (for further discussion, see Lauritzen, 2001; Logothetis et al., 2001, 2008; Vanzetta and Grinvald, 2008).

$+0 \mathrm{~h}$ animals: baseline level initial dip recovers within $90 \mathrm{~min}$ of treatment and baseline level overshoot recovers within 120 $\min$

For $+0 \mathrm{~h}$ animals, postocclusion ISOI revealed differences across time for both the area $\left(F_{(3,27)}=24.06, p<0.01\right.$, ANOVA $)$ and amplitude $\left(F_{(3,27)}=24.06, p<0.01\right.$, ANOVA $)$ of the initial dip. When compared with baseline imaging, the area was reduced at the first time point $\left(0-30 \mathrm{~min}\right.$ of stimulation treatment; $F_{(1,9)}=$ $14.93, p<0.01)$ and at the second time point $(30-60 \mathrm{~min}$ of treatment; $\left.F_{(1,9)}=10.10, p<0.05\right)$. The amplitude was also reduced compared with baseline for $0-30 \mathrm{~min}\left(F_{(1,9)}=14.75\right.$, $p<0.01)$ and for $30-60 \min \left(F_{(1,9)}=9.255, p<0.05\right)$. By $90 \mathrm{~min}$ of treatment, the initial dip had regained baseline values for both area and amplitude (Fig. $2 A$, green).

Similar to the initial dip phase data, there were differences across time for both the area $\left(F_{(3,27)}=11.29, p<0.01\right.$, ANOVA $)$ and amplitude $\left(F_{(3,27)}=14.32, p<0.01\right.$, ANOVA $)$ of the over-

Figure 3. Vital parameters remain stable throughout the treatment period in protected subjects. In each plot, means and SEs are provided for heart rate, breath rate, arterial saturation, and pulse distension (blood pressure) before and after pMCAO in $+0 \mathrm{~h}$ subjects $(n=10)$; all four parameters remained stable throughout the duration of the experiment.

shoot phase. The overshoot area was reduced from $0-30 \mathrm{~min}$ $\left(F_{(1,9)}=19.00, p<0.01\right)$ and $30-60 \min \left(F_{(1,9)}=14.62, p<\right.$ 0.01 ), but regained baseline level area by 90 min of treatment. The overshoot amplitude, however, required an additional $30 \mathrm{~min}$ of treatment before it regained pre-pMCAO levels. The amplitude was reduced from $0-30\left(F_{(1,9)}=22.1, p<0.01\right), 30-60\left(F_{(1,9)}=\right.$ 8.77, $p<0.05)$, and $60-90 \mathrm{~min}\left(F_{(1,9)}=8.38, p<0.05\right)$ of treatment (Fig. $2 B$, green).

To ensure that the observed protection was not due to an alteration of vital parameters such as blood pressure, arterial $\mathrm{O}_{2}$ saturation, respiration, or heart rate resulting from the whisker stimulation, we conducted an additional series of $+0 \mathrm{~h}(n=10)$ experiments during which we monitored these parameters using a pulse-oximetry system; all four parameters remained stable throughout the duration of the experiment [heart rate, $F_{(36,324)}=$ $0.881, p>0.05$; respiration rate, $F_{(36,324)}=0.592, p>0.05$; pulse distension (a proxy measurement for blood pressure), $F_{(36,324)}=$ $0.946, p>0.05$; arterial saturation, $F_{(36,324)}=1.423 p>0.05$; within-subjects ANOVAs] (Fig. 3).

\section{$+1 \mathrm{~h}$ animals: baseline level initial dip and overshoot recover} within 90 min of treatment

$+1 \mathrm{~h}$ group results were similar to those observed with the $+0 \mathrm{~h}$ group. Postocclusion imaging revealed differences across time for both the area $\left(F_{(3,27)}=4.30, p<0.01\right.$, ANOVA $)$ and amplitude $\left(F_{(3,27)}=8.873, p<0.01\right.$, ANOVA $)$ of the initial dip. The area was reduced compared with baseline from $0-30 \mathrm{~min}$ $\left(F_{(1,9)}=26.90, p<0.01\right)$ and $30-60 \min \left(F_{(1,9)}=24.96, p<\right.$ $0.01)$ of treatment. The initial dip amplitude was also reduced compared with baseline from $0-30 \min \left(F_{(1,9)}=28.21, p<0.01\right)$ and $30-60 \mathrm{~min}\left(F_{(1,9)}=11.37, p<0.01\right)$. By 90 min of treatment, the initial dip had regained baseline values for both area and amplitude (Fig. $2 A$, blue).

$+1 \mathrm{~h}$ group overshoot shared the same time course of return as the initial dip: there were differences across time for both the area $\left(F_{(3,27)}=9.0, p<0.01\right.$, ANOVA $)$ and amplitude $\left(F_{(3,27)}=\right.$ $12.13, p<0.01$, ANOVA). Overshoot area was reduced from $0-30 \min \left(F_{(1,9)}=8.99, p<0.05\right)$ and $30-60 \min \left(F_{(1,9)}=12.79\right.$, 
$p<0.01)$ of treatment and the amplitude was also reduced from $0-30\left(F_{(1,9)}=46.14, p<0.01\right)$ and $30-60 \min \left(F_{(1,9)}=18.29\right.$, $p<0.01$ ) (Fig. 2 B, blue). By 90 min of treatment, the overshoot had also regained baseline values for both area and amplitude.

$+2 \mathrm{~h}$ animals: in protected $+2 \mathrm{~h}$ animals ( 7 of 10$)$, baseline level initial dip and overshoot recovered within $90 \mathrm{~min}$ of treatment

Though most $+2 \mathrm{~h}$ animals (7 of 10) recovered baseline level whisker functional representation by $90 \mathrm{~min}$ of stimulation treatment (a similar recovery time course to $+0 \mathrm{~h}$ and $+1 \mathrm{~h}$ subjects) and showed no sign of infarct, three animals exhibited some evidence of a stroke. These three partially protected $+2 \mathrm{~h}$ animals had whisker functional representation values that remained below baseline throughout treatment. These three animals also sustained infarct (though this infarct was smaller than that observed in $+3 \mathrm{~h}$ animals; range, $15.4-35.0 \mathrm{~mm}^{3}$; mean, $24.2 \pm 5.8 \mathrm{~mm}^{3}$ ). Though noticeably reduced, whisker functional representations in partially protected $+2 \mathrm{~h}$ subjects were not absent. Both the initial dip and overshoot remained present, but never regained pre-pMCAO baseline levels during the treatment period (data not shown). Because the outcomes between protected and partially protected $+2 \mathrm{~h}$ subjects were qualitatively very different, we chose to segregate the data into protected and partially protected $+2 \mathrm{~h}$ animal subsets for data analysis.

In protected $+2 \mathrm{~h}$ animals, postocclusion imaging revealed differences across time for both the area $\left(F_{(3,18)}=10.56, p<\right.$ 0.001 , ANOVA) and amplitude $\left(F_{(3,18)}=6.36, p<0.01\right.$, ANOVA) of the initial dip. The area was reduced compared with baseline for $0-30 \min \left(F_{(1,6)}=35.71, p<0.001\right)$ and for $30-60$ $\min \left(F_{(1,6)}=27.42, p<0.01\right)$ of treatment. The amplitude was also reduced compared with baseline for $0-30 \mathrm{~min}\left(F_{(1,6)}=\right.$ $14.43, p<0.01)$ of treatment. By 90 min of treatment, the initial dip had regained baseline values for both area and amplitude (Fig. 2A, orange).

There were also differences across time for both the overshoot area $\left(F_{(3,18)}=6.81, p<0.01\right.$, ANOVA $)$ and amplitude $\left(F_{(3,18)}=\right.$ $7.15, p<0.01$, ANOVA). Overshoot area was reduced compared with baseline for $0-30 \mathrm{~min}\left(F_{(1,6)}=173.54, p<0.001\right)$ and for $30-60 \min \left(F_{(1,6)}=29.40, p<0.01\right)$ of treatment. The amplitude was also reduced compared with baseline for $0-30 \mathrm{~min}\left(F_{(1,6)}=\right.$ 22.79, $p<0.01)$ and for $30-60 \min \left(F_{(1,6)}=14.21, p<0.01\right)$ of treatment (Fig. $2 B$, orange). By 90 min of treatment, the overshoot had also regained baseline values for both area and amplitude.

\section{Functional recovery within the ischemic cortex is possible after an extended $(2 \mathrm{~h})$ delay but neither pMCAO nor treatment alter contra-ischemic function}

Finally, we conducted a between-groups analysis of recovery to determine explicitly whether any differences in whisker functional representation exist between protected $+0 \mathrm{~h},+1 \mathrm{~h}$, and +2 $\mathrm{h}$ animals. All groups were assessed at baseline and at four time points (spaced $\sim 30 \mathrm{~min}$ apart) across the $120 \mathrm{~min}$ treatment period. There were no significant between-group differences in either the initial dip (area, $F_{(2,24)}=0.16, p>0.05$; amplitude, $F_{(2,24)}=0.14, p>0.05$; between-subjects ANOVAs) or the overshoot (area, $F_{(2,24)}=0.18, p>0.05$; amplitude, $F_{(2,24)}=1.99, p>$ 0.05; between-subjects ANOVAs) throughout experimentation.

Together, these data demonstrate that, when delivered either 0,1 , or $2 \mathrm{~h}$ postocclusion, whisker stimulation treatment initiates a similar reestablishment time course profile for whisker functional representation, both in terms of the initial dip and the overshoot phases of the signal. By 90 min of treatment, both the
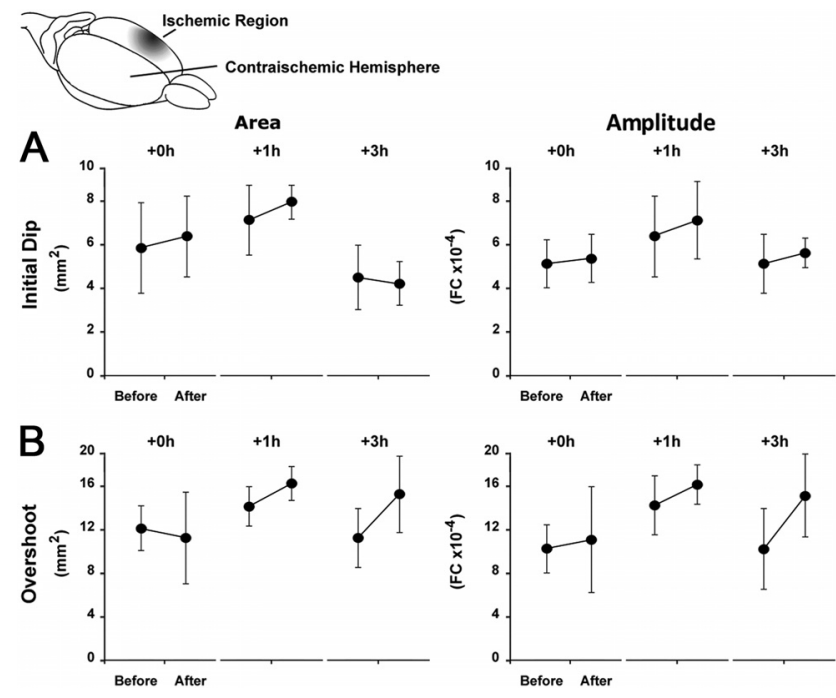

Figure 4. Contra-ischemic ISOI reveals that changes in cortical function are limited to the ischemic hemisphere. The initial dip and overshoot phases of the contra-ischemic whisker functional representation remain constant throughout experimentation. $\boldsymbol{A}, \boldsymbol{B}$, Group baseline is paired with postocclusion stimulus data. Means and SEs are provided for the area (left) and amplitude (right) of the contra-ischemic C 2 initial dip ( $A$ ) and overshoot (B) phases of whisker functional representation before and immediately after stimulation treatment. ANOVA confirmed that there were no differences before and after pMCAO occlusion for either the area $\left(+0 \mathrm{~h}: F_{(1,6)}=0.0004, p>0.05 ;+1 \mathrm{~h}: F_{(1,6)}=0.078, p>\right.$ $\left.0.05 ;+3 \mathrm{~h}: F_{(1,6)}=0.007, p>0.05\right)$ and amplitude $\left(+0 \mathrm{~h}: F_{(1,6)}=0.009, p>0.05 ;+1\right.$ $\left.\mathrm{h}: F_{(1,6)}=0.275, p>0.05 ;+3 \mathrm{~h}: F_{(1,6)}=0.132, p>0.05\right)$ of the initial dip, or the area $\left(+0 \mathrm{~h}: F_{(1,6)}=0.044, p>0.05 ;+1 \mathrm{~h}: F_{(1,6)}=0.215, p>0.05 ;+3 \mathrm{~h}: F_{(1,6)}=1.252\right.$, $p>0.05)$ and amplitude $\left(+0 \mathrm{~h}: F_{(1,6)}=0.027, p>0.05 ;+1 \mathrm{~h}: F_{(1,6)}=0.729, p>\right.$ $\left.0.05 ;+3 \mathrm{~h}: F_{(1,6)}=0.912, p>0.05\right)$ of the overshoot phase. FC, Fractional change.

area and amplitude of the initial dip had returned to preocclusion levels in all three groups. The overshoot area was reestablished after 90-120 min of treatment in all three groups (overshoot amplitude required 120 min of treatment to reach baseline in +0 $h$ animals).

Finally, we previously reported that the contra-ischemic cortex was unchanged from baseline in all animals, regardless of experimental group at $24 \mathrm{~h}$ post-pMCAO (Lay et al., 2010). Here we follow up this finding in $+0 \mathrm{~h},+1 \mathrm{~h}$, and $+3 \mathrm{~h}$ experimental groups ( $n=7$ per group) by verifying that evoked cortical function in the contra-ischemic hemisphere remained unchanged immediately following the treatment period, suggesting that the differences between experimental groups were limited to the ischemic hemisphere (Fig. 4; see legend for details and statistical analyses).

Evoked neuronal activity is severely reduced by pMCAO and recovers to baseline or near-baseline levels within $120 \mathrm{~min}$ of treatment

We next sought to determine the impact of protective stimulation treatment on evoked subthreshold and suprathreshold neuronal activity in the cortex. LFP and MUA were quantified in the supragranular layer (300-400 $\mu \mathrm{m}$ below the cortical surface) of the cortex at the location of peak ISOI activity in $+0 \mathrm{~h}$ animals $(n=9)$ before pMCAO and at four time points (spaced $\sim 30 \mathrm{~min}$ apart) during the $120 \mathrm{~min}$ treatment period (Fig. 5). Simultaneous recording revealed differences across time for both LFP $\left(F_{(4,32)}=20.75, p<0.01\right.$, ANOVA $)$ and $\operatorname{MUA}\left(F_{(4,32)}=14.95\right.$, $p<0.01$, ANOVA). There was a loss of neuronal response following pMCAO that was characterized by a large reduction in the magnitude of LFP and MUA (to noise or near-noise levels). Dur- 


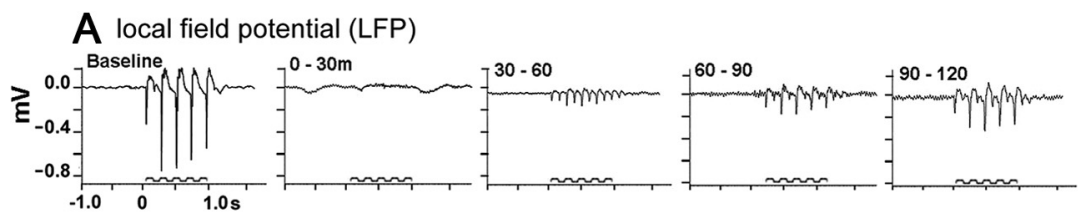

B multi unit activity (MUA)
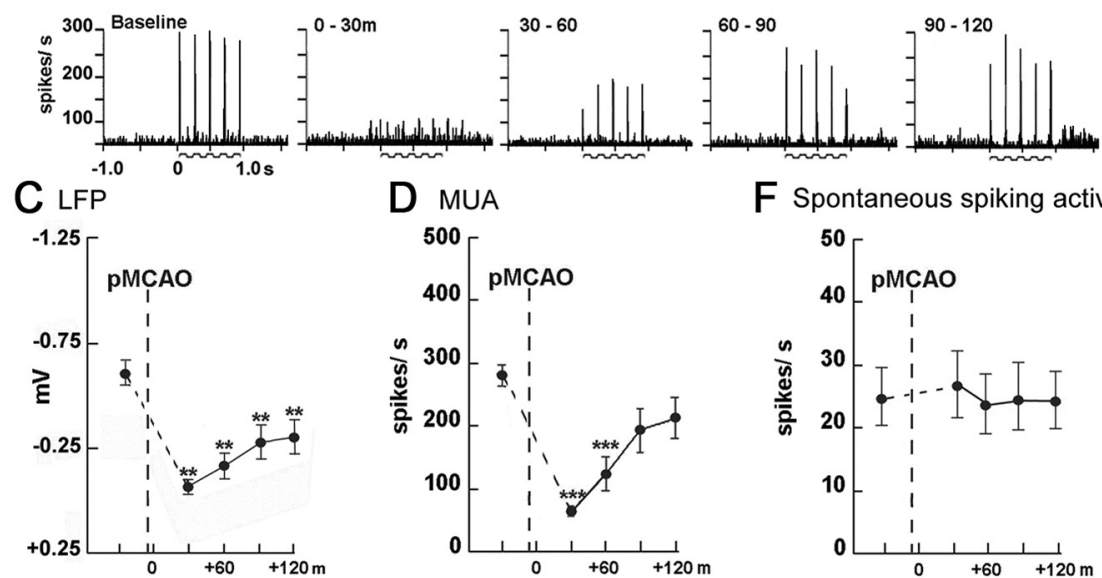

F spontaneous spiking activity

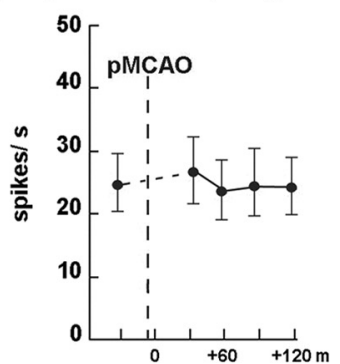

E MUA raster plot

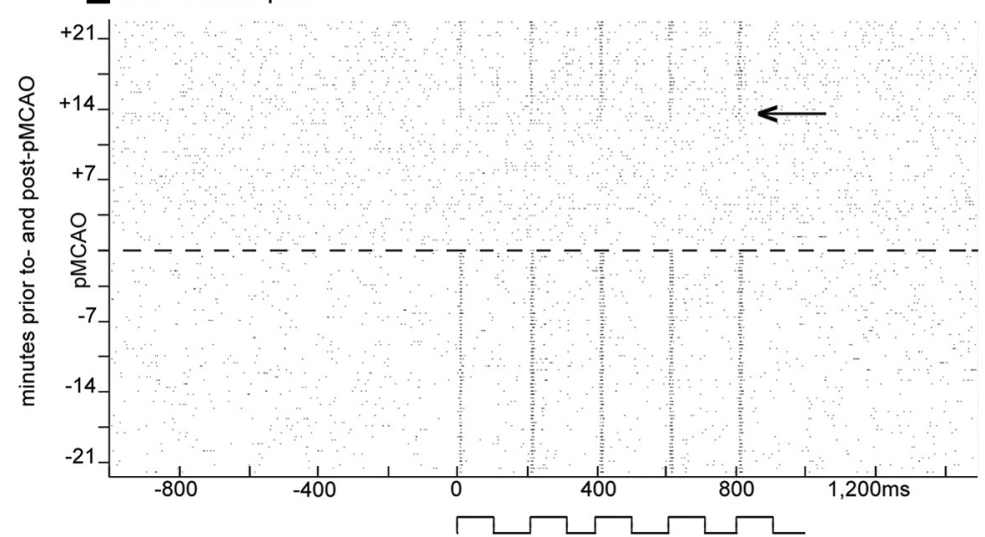

Figure 5. Evoked neuronal activity was suppressed following $\mathrm{PMCAO}$ and reestablished during whisker stimulation treatment in $+0 \mathrm{~h}$ animals. $\boldsymbol{A}, \boldsymbol{B}$, Representative $+0 \mathrm{~h}$ animals ${ }^{\prime} \operatorname{LFP}(\boldsymbol{A})$ and MUA $(\boldsymbol{B})$ responses at baseline and during the stimulus period following pMCA0. Stepping function indicates stimulus delivery. $\boldsymbol{C}, \boldsymbol{D}, \boldsymbol{F}$, Evoked LFP $(\boldsymbol{C})$, evoked MUA (D), and spontaneous spiking activity $(\boldsymbol{F})$ mean and $\mathrm{SE}$ is plotted preocclusion and postocclusion. Asterisks indicated a significant difference from baseline: ${ }^{* *} p<0.01$ and ${ }^{* * *} p<0.001$. E, Representative multiunit raster plot recorded $\sim 21 \mathrm{~min}$ before and after pMCA0 ( $\sim 21$ min are required to collect one full 64 stimulus trial block). $x$-Axis represents $1000 \mathrm{~ms}$ of spontaneous activity, $1000 \mathrm{~ms}$ of evoked activity (whisker stimulation indicated by step function), and $500 \mathrm{~ms}$ of spontaneous activity. Arrow indicates the approximate point at which evoked MUA responses begin to return.

ing treatment, we observed a gradual return in LFP activity, though it remained below baseline levels throughout the treatment period (Fig. $5 A, C$ ). MUA was also severely reduced postocclusion (Fig. $5 B, D$ ), remained depressed at the $0-30$ and $30-60$ min time points $\left(0-30 \mathrm{~min}: F_{(1,8)}=11.48, p<0.001\right.$; 30-60 min: $\left.F_{(1,8)}=42.77, p<0.001\right)$ and, similar to whisker functional representation phases, returned to baseline levels by 90 min of treatment. When assessed on a single trial level, evoked spiking activity (MUA) disappeared completely in response to pMCAO and began to gradually return after $17.5 \pm 4.5 \mathrm{~min}$ of treatment (mean \pm SEM; range, $2-40 \mathrm{~min}$ ) in nine of nine $+0 \mathrm{~h}$ subjects (for representative case, see Fig. $5 F$ ). Because stimulation over the treatment period of $17.5 \mathrm{~min}$ is intermittent, only $\sim 50$ total seconds of stimulation were delivered before evoked spiking began to return. Once reinitiated, MUA remained present and increased until reaching baseline levels of activity within 60-90 min of treatment.

Interestingly, spontaneous spiking activity remained at the same pre-pMCAO level throughout the entire experiment (Fig. $5 E$ ), regardless of pMCAO or stimulation treatment $\left(F_{(4,32)}=0.15, p>0.05\right.$, ANOVA).

\section{Perfusion is severely reduced by pMCAO and reperfusion is initiated by stimulation and recovers gradually during treatment}

To characterize reperfusion induced by whisker stimulation treatment in the ischemic cortex (presumably a prerequisite for its protection), laser speckle imaging of blood flow before pMCAO and at four time points (spaced $\sim 30 \mathrm{~min}$ apart) during the 120 min treatment period was also performed in a separate group of $+0 \mathrm{~h}$ animals $(n=7)$ and compared with a no-stimulus control group $(n=6)$ that underwent pMCAO but never received whisker stimulation treatment. In both groups, we observed a substantial decrease in perfusion within MCA cortical branches following pMCAO. This was followed by a gradual increase in perfusion in the $+0 \mathrm{~h}$ group but not in the no-stimulus control group during the $120 \mathrm{~min}$ treatment period. Laser speckle imaging of MCA demonstrated that perfusion dropped substantially in both groups following occlusion (to $26 \pm 4 \%$ of baseline values) (Fig. 6) and then increased in the +0 $\mathrm{h}$ group throughout stimulation treatment compared with the no-stimulus controls $\left(F_{(1,11)}=5.48, p<0.05\right.$, ANOVA), which did not change after dropping postpMCAO. By the completion of 120 min of treatment, $+0 \mathrm{~h}$ values were significantly above the no-stimulus control group values $\left(F_{(1,11)}=10.87, p<0.01\right)$ and had reached $51 \pm 5 \%$ of baseline levels.

Animals subjected to $+0 \mathrm{~h}$ protocol were never observed to sustain infarct, while no-stimulus controls sustained consistent infarct volumes averaging $28.4 \pm 2.4 \mathrm{~mm}^{3}$ (Lay et al., 2010).

\section{Discussion}

The brain's capacity for reorganization underlies adaptive responses to environmental stimulation and even rehabilitation after neurological injuries in the adult cortex (Greenberg and Jin, 2007; Jäncke, 2009). The mild-stimulation-initiated protection observed in this study raises hope for the development of stimulation-based strategies by which such dynamic brain processes might be used to mitigate or eradicate stroke pathology in humans. We have previously shown that the cortex is functionally and structurally intact $24 \mathrm{~h}$ post-pMCAO when stimulation treatment is administered within 1 and in most cases $2 \mathrm{~h}$ of ischemic onset. 


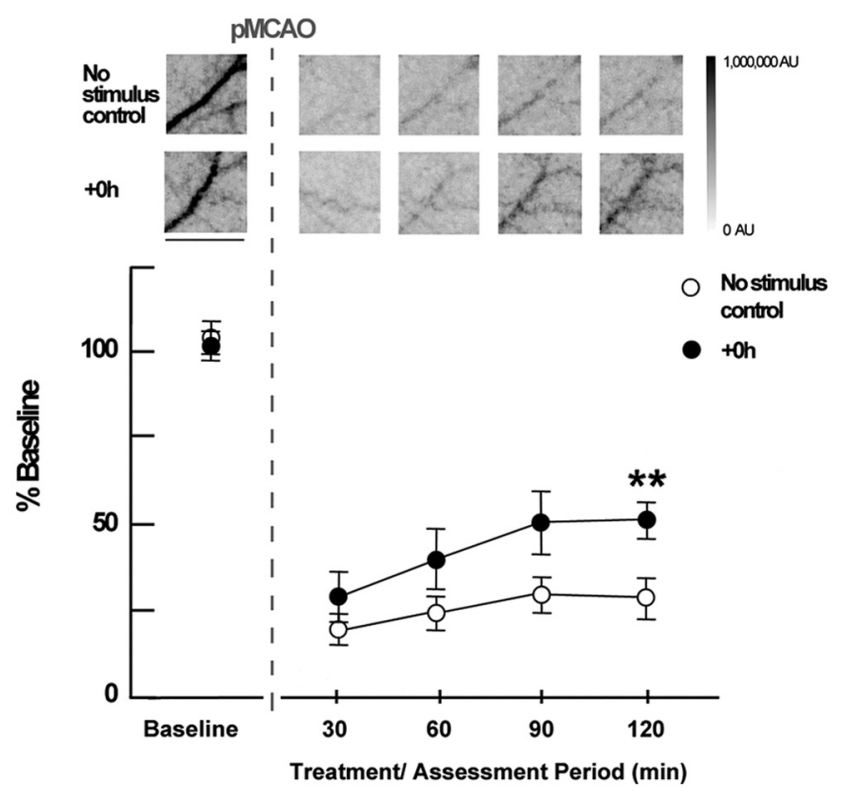

Figure 6. LSI experiments demonstrate that post-pMCAO blood flow return in MCA is induced by whisker stimulation treatment. Insets, Representative cases of no-stimulus control and $+0 \mathrm{~h}$ animals presented as linearly gray-scaled $\mathrm{LS}$ images taken at baseline and following pMCAO during treatment at $\sim 30$ min intervals. Scale bar, $1 \mathrm{~mm}$. The dark vessel diagonally traversing the image in each case is a cortical branch of MCA (most clear in preocclusion baseline images; other vessels such as dural vessels are also visible, but much less apparent than MCA at baseline). Representative segments of MCA branch ROls above the region of somatosensory cortex, distal to PMCAO are shown [(for an in depth description regarding criteria for MCA regions of interest analyzed, see the Methods section of Lay et al. (2010)]. Means and SEs for MCA for no-stimulus and $+0 \mathrm{~h}$ animals at baseline, following pMCA0, and during whisker stimulation. ${ }^{* *} p<0.01$, significant increase in $+0 \mathrm{~h}$ flow compared with the no-stimulus control group.

Here, we assess the effect of this stimulation acutely, during treatment. We show that recovery is initiated by treatment onset and that evoked responses and reperfusion during treatment period can be used to predict outcome.

\section{Gradual recovery is initiated with treatment onset in all protected animals and is predictive of outcome}

Functional imaging of evoked responses in the ischemic cortex during stimulation revealed that recovery was not initiated until the onset of stimulation regardless of when it was delivered $(0,1$, or $2 \mathrm{~h}$ postocclusion). Unprotected animals' $(+3 \mathrm{~h})$ responses were completely absent at all postocclusion time points; in partially protected $+2 \mathrm{~h}$ animals (3 of 10 animals), the signal was severely reduced at all time points. For all groups, acute period results were predictive of $24 \mathrm{~h}$ results, including infarct or lack thereof.

Although the WFR in $+0 \mathrm{~h},+1 \mathrm{~h}$, and $+2 \mathrm{~h}$ groups shared a similar recovery pattern overall, in some cases the overshoot amplitude did not fully recover until $30 \mathrm{~min}$ after the initial dip amplitude. We have previously demonstrated that the spatiotemporal characteristics of these phases are generally independent in healthy animals (Chen-Bee et al., 2007), so the differential is not entirely surprising, but if an across-phase reduction in WFR alone were the cause for the difference, one would expect the signals to return at the same rate. This result suggests that there is an alteration in the hemodynamic response during the recovery phase.

While all $+0 \mathrm{~h}$ and $+1 \mathrm{~h}$ subjects recovered fully, three of 10 $+2 \mathrm{~h}$ subjects did not. These three $+2 \mathrm{~h}$ subjects sustained cor- tical infarct, but of a smaller volume (range, $15.4-35.0 \mathrm{~mm}^{3}$; mean, $24.2 \pm 5.8 \mathrm{~mm}^{3}$ ) than that of $+3 \mathrm{~h}$ subjects (range, 30.4$82.6 \mathrm{~mm}^{3}$; mean, $59.2 \pm 4.4 \mathrm{~mm}^{3}$ ). Additionally, while the unprotected $+3 \mathrm{~h}$ subjects displayed no WFR at any postocclusion time point, the partially protected $+2 \mathrm{~h}$ animals maintained a diminished but present WFR. These data likely indicate that there exists a transition between 2 and $3 \mathrm{~h}$ after ischemic onset during which stimulation treatment goes from being completely protective, to partially protective, and finally to being harmful [our previous study showed that stimulation at $3 \mathrm{~h}$ post-pMCAO caused more damage than no stimulation after pMCAO (Lay et al., 2010)].

\section{Protection is conferred by a stimulation-induced gradual reperfusion that does not occur spontaneously}

Previous research demonstrated that protected animals have baseline level blood flow in MCA (via collaterals) at $24 \mathrm{~h}$ postpMCAO (Lay et al., 2010). What was unclear, however, was whether collateral reperfusion was spontaneous or initiated by stimulation treatment. Based on the current results, the latter is the case. $+0 \mathrm{~h}$ animals' flow levels increase gradually from stimulation onset and are significantly different from no-stimulus controls by $120 \mathrm{~min}$ post-pMCAO. This reperfusion did not occur in no-stimulus control animals that underwent pMCAO without stimulation treatment (and sustained infarct)-their flow levels remained at post-pMCAO drop levels.

It is surprising that, despite the dramatic and prolonged drop in blood flow resulting from pMCAO (to $\sim 26 \%$ of baseline according to LSI), most $+2 \mathrm{~h}$ animals are completely protected from damage. Given that blood flow drops to a level considered to be the threshold for irreversible damage (Hossmann, 1994; Iadecola and Nedergaard, 2007) and does not spontaneously increase, $+2 \mathrm{~h}$ animals' are in a severely ischemic state for two full hours before stimulation is initiated, and yet this treatment can still save the cortex. Burnett and colleagues (2006) induced a temporary MCAO that caused a drop in blood flow (to $26-27 \%$ of baseline according to laser Doppler flowmetry) similar to our pMCAO (which causes a drop to $\sim 26 \%$ according to LSI) [measurements obtained using laser Doppler flowmetry and LSI data have been shown to be highly correlated (Dunn et al., 2001; Choi et al., 2006)]. Burnett et al. (2006) also delivered a stimulation treatment (electrical forepaw stimulation), but in addition released the temporary occlusion after $1.5 \mathrm{~h}$, allowing reperfusion to a level of $110 \%$ of baseline. This resulted in a decrease but not elimination of infarct and no reduction in behavioral deficits. It may be that the relatively slow collateral-based blood flow return (like that induced by sensory stimulation in our study) is a preferable means of reperfusion over sudden and strong return of flow (like that resulting from removal of a temporary occlusion). If gradual reperfusion is preferable to sudden and strong reperfusion, it would explain why animals in our study are completely protected. This may also shed light on necessary components of future clinical stroke treatments with reference to how reperfusion is achieved within the critical time window.

Molecular and cellular mechanisms for the induction of reperfusion in our study may be similar to those involved in healthy activation flow coupling in the brain, but require more research to be determined. In addition, the mechanism by which blood flow is maintained and increased from $50 \%$ (at the completion of stimulation treatment) to $\sim 100 \%$ by the following day remains to be determined. 


\section{Comparison of the time course of recovery of whisker functional representation, neuronal responses, and reperfusion}

Evoked suprathreshold responses (MUA) were eliminated or nearly eliminated following pMCAO, but began to recover 17.5 min into treatment on average (at this point, only $50 \mathrm{~s}$ of whisker stimulation had been delivered) and were at baseline level by 90 $\min$ (3.2 min of whisker stimulation). Interestingly, this timing of recovery is similar to the return of the area and amplitude of WFR in protected animals.

Subthreshold-evoked responses also dropped to near-noise immediately following pMCAO and, although there was also a gradual increase as stimulation progressed, values remained below baseline throughout the treatment period. Given that suprathreshold activity is thought to contribute to the initial dip phase of the WFR and that current research reflects a growing assertion that subthreshold activity may be more critical in driving evoked hemodynamic responses (Lauritzen, 2001, 2005; Thomsen et al., 2004), it seems reasonable that the initial dip versus overshoot return observed in this study might be a result of neuronal recovery occurring ahead of a full reperfusion. Indeed, our LSI data also suggest that reperfusion recovery better matches the slower recovery of the overshoot phase of the WFR: immediately following pMCAO, blood flow dropped to $26 \%$ of baseline and then returned gradually in protected animals but also did not reach baseline levels during treatment [both subthreshold responses and blood flow were shown to be at preocclusion levels by $24 \mathrm{~h}$ post-pMCAO in the same animal model in Lay et al. (2010)]. Additionally, if suprathreshold responses are not as well linked to blood flow responses, it seems reasonable to assume that full recovery of spiking responses could occur before full recovery of blood flow. These comparisons were made between animals, however, as electrophysiological and functional imaging experiments were performed independently.

Interestingly, despite the drastic drop in evoked signals and blood flow in response to pMCAO, spontaneous spiking activity remained constant (at pre-pMCAO levels) throughout the experiment. These results suggest that while evoked spiking is impaired by ischemic conditions, neurons within the barrel cortex are capable of firing action potentials, even immediately following pMCAO when perfusion of the cortex is at its lowest point. Thus, the ability of neurons to spontaneously fire was not affected by ischemia, but the ability of stimulation to evoke firing was affected. Despite the fact that stimulation during the early treatment period was not able to evoke spiking responses in the ischemic cortex, its administration is what facilitated the return of this ability. Clearly, there is still much research to be done regarding the roles of various types of activation in the ischemic cortex.

This study characterized the acute effect of protective whisker stimulation treatment during ischemia. Further studies are still needed to unravel the molecular and cellular mechanisms by which mild, noninvasive sensory stimulation induces reperfusion, restoration of function, and prevention of structural damage and to characterize what types of stimulation can be effective. The current study also suggests that there exist diagnostic markers predictive of outcome during stimulation treatment in the acute phase of ischemia; protected and partially protected animals were readily distinguishable from unprotected animals based on evoked hemodynamic signal as soon as $60 \mathrm{~min}$ after treatment had begun. Thus, further sensory stimulation-induced protection studies could lead to the development of rapid stroke interventions that could be assessed for their effectiveness during treatment itself and could potentially be administered to a wider range of patients without side effects.

\section{References}

Armitage GA, Todd KG, Shuaib A, Winship IR (2010) Laser speckle contrast imaging of collateral blood flow during acute ischemic stroke. J Cereb Blood Flow Metab 30:1432-1436.

Bederson JB, Pitts LH, Tsuji M, Nishimura MC, Davis RL, Bartkowski H (1986) Rat middle cerebral artery occlusion: evaluation of the model and development of a neurologic examination. Stroke 17:472-476.

Brett-Green BA, Chen-Bee CH, Frostig RD (2001) Comparing the functional representations of central and border whiskers in rat primary somatosensory cortex. J Neurosci 21:9944-9954.

Briers JD, Webster S (1995) Quasi real-time digital version of singleexposure photography for full-field monitoring of velocity or flow-fields. Opt Commun 36-42.

Brint S, Jacewicz M, Kiessling M, Tanabe J, Pulsinelli W (1988) Focal brain ischemia in the rat: methods for reproducible neocortical infarction using tandem occlusion of the distal middle cerebral and ipsilateral common carotid arteries. J Cereb Blood Flow Metab 8:474-485.

Burnett MG, Shimazu T, Szabados T, Muramatsu H, Detre JA, Greenberg JH (2006) Electrical forepaw stimulation during reversible forebrain ischemia decreases infarct volume. Stroke 37:1327-1331.

Chen-Bee CH, Kwon MC, Masino SA, Frostig RD (1996) Areal extent quantification of functional representations using intrinsic signal optical imaging. J Neurosci Methods 68:27-37.

Chen-Bee CH, Polley DB, Brett-Green B, Prakash N, Kwon MC, Frostig RD (2000) Visualizing and quantifying evoked cortical activity assessed with intrinsic signal imaging. J Neurosci Methods 97:157-173.

Chen-Bee CH, Agoncillo T, Xiong Y, Frostig RD (2007) The triphasic intrinsic signal: implications for functional imaging. J Neurosci 27:4572-4586.

Choi B, Ramirez-San-Juan JC, Lotfi J, Stuart Nelson J (2006) Linear response range characterization and in vivo application of laser speckle imaging of blood flow dynamics. J Biomed Opt 11:041129.

Davis MF, Lay CC, Chen-Bee CH, Frostig RD (2011) Amount but not pattern of protective sensory stimulation alters recovery after permanent middle cerebral artery occlusion. Stroke 42:792-798.

Dunn AK, Bolay H, Moskowitz MA, Boas DA (2001) Dynamic imaging of cerebral blood flow using laser speckle. J Cereb Blood Flow Metab 21:195-201

Fox G, Gallacher D, Shevde S, Loftus J, Swayne G (1993) Anatomic variation of the middle cerebral artery in the Sprague-Dawley rat. Stroke 24:20872092; discussion 2092-2093.

Frostig RD, Chen-Bee CH (2009) Visualizing adult cortical plasticity using intrinsic signal optical imaging. In: In vivo optical imaging of brain function (Frostig RD, ed), pp 255-287. Boca Raton, FL: CRC.

Frostig RD, Lieke EE, Ts'o DY, Grinvald A (1990) Cortical functional architecture and local coupling between neuronal activity and the microcirculation revealed by in vivo high-resolution optical imaging of intrinsic signals. Proc Natl Acad Sci U S A 87:6082-6086.

Frostig RD, Xiong Y, Chen-Bee CH, Kvasnák E, Stehberg J (2008) Largescale organization of rat sensorimotor cortex based on a motif of large activation spreads. J Neurosci 28:13274-13284.

Goldlust EJ, Paczynski RP, He YY, Hsu CY, Goldberg MP (1996) Automated measurement of infarct size with scanned images of triphenyltetrazolium chloride-stained rat brains. Stroke 27:1657-1662.

Greenberg DA, Jin K (2007) Regenerating the brain. Int Rev Neurobiol 77:1-29.

Grinvald A, Lieke E, Frostig RD, Gilbert CD, Wiesel TN (1986) Functional architecture of cortex revealed by optical imaging of intrinsic signals. Nature 324:361-364.

Hossmann KA (1994) Viability thresholds and the penumbra of focal ischemia. Ann Neurol 36:557-565.

Iadecola C, Nedergaard M (2007) Glial regulation of the cerebral microvasculature. Nat Neurosci 10:1369-1376.

Jäncke L (2009) The plastic human brain. Restor Neurol Neurosci 27: 521-538.

Lauritzen M (2001) Relationship of spikes, synaptic activity, and local changes of cerebral blood flow. J Cereb Blood Flow Metab 21:1367-1383.

Lauritzen M (2005) Reading vascular changes in brain imaging: is dendritic calcium the key? Nat Rev Neurosci 6:77-85. 
Lavie G, Teichner A, Shohami E, Ovadia H, Leker RR (2001) Long term cerebroprotective effects of dexanabinol in a model of focal cerebral ischemia. Brain Res 901:195-201.

Lay CC, Davis MF, Chen-Bee CH, Frostig RD (2010) Mild sensory stimulation completely protects the adult rodent cortex from ischemic stroke. PLoS One 5:e11270.

Lloyd-Jones D, Adams R, Carnethon M, De Simone G, Ferguson TB, Flegal K, Ford E, Furie K, Go A, Greenlund K, Haase N, Hailpern S, Ho M, Howard V, Kissela B, Kittner S, Lackland D, Lisabeth L, Marelli A, McDermott M, et al. (2009) Heart disease and stroke statistics-2009 update: a report from the American Heart Association Statistics Committee and Stroke Statistics Subcommittee. Circulation 119:e21-e181.

Logothetis NK (2008) What we can do and what we cannot do with fMRI. Nature 453:869-878.

Logothetis NK, Pauls J, Augath M, Trinath T, Oeltermann A (2001) Neurophysiological investigation of the basis of the fMRI signal. Nature 412:150-157.

Masino SA, Kwon MC, Dory Y, Frostig RD (1993) Characterization of functional organization within rat barrel cortex using intrinsic signal optical imaging through a thinned skull. Proc Natl Acad Sci U S A 90:999810002.

Murphy TH, Corbett D (2009) Plasticity during stroke recovery: from synapse to behaviour. Nat Rev Neurosci 10:861-872.
Niiro M, Simon RP, Kadota K, Asakura T (1996) Proximal branching patterns of middle cerebral artery (MCA) in rats and their influence on the infarct size produced by MCA occlusion. J Neurosci Methods 64:19-23.

Schaffer CB, Friedman B, Nishimura N, Schroeder LF, Tsai PS, Ebner FF, Lyden PD, Kleinfeld D (2006) Two-photon imaging of cortical surface microvessels reveals a robust redistribution in blood flow after vascular occlusion. PLoS Biol 4:e22.

Tamura A, Graham DI, McCulloch J, Teasdale GM (1981) Focal cerebral ischaemia in the rat. 1. Description of technique and early neuropathological consequences following middle cerebral artery occlusion. J Cereb Blood Flow Metab 1:53-60.

Thomsen K, Offenhauser N, Lauritzen M (2004) Principal neuron spiking: neither necessary nor sufficient for cerebral blood flow in rat cerebellum. J Physiol 560:181-189.

Ts'o DY, Frostig RD, Lieke EE, Grinvald A (1990) Functional organization of primate visual cortex revealed by high resolution optical imaging. Science 249:417-420.

Vanzetta I, Grinvald A (2008) Coupling between neuronal activity and microcirculation: implications for functional brain imaging. Hfsp J 2:79-98.

Wang-Fischer Y (2009) Manual of stroke models in rats. Boca Raton, FL: CRC. 